\title{
Synthesis of donor-acceptor substituted oligothiophenes by Stille coupling
}

\author{
M. Manuela M. Raposo*a A. Maurício C. Fonseca ${ }^{\mathrm{a}}$ and G. Kirsch ${ }^{\mathrm{b}}$ \\ a Centro de Química, Universidade do Minho, Campus de Gualtar \\ 4710-057 Braga, Portugal, e-mail mfox@quimica.uminho.pt \\ b Laboratoire d'Ingénierie Moléculaire et Biochimie Pharmacologique, \\ UFR SciFA/Université de Metz, 1, bd Arago, Metz Technopôle, CP 87811 \\ 57078 Metz Cedex 3, France
}

\begin{abstract}
A synthesis of donor-acceptor-substituted oligothiophenes by Stille coupling is described. The 5 '-estanyl derivatives, readily prepared from 5 -alkoxy- and 5-amino2,2'-bithiophenes 7 were coupled with the appropriate aryl or heteroaryl bromides to give the title compounds.
\end{abstract}

Keywords: 5-alkoxy- and 5-amino-2,2'-bithiophenes, Stille coupling, donor-acceptor oligothiophenes, UV-visible spectroscopy, chromophores, solvatochromism, non-linear optical (NLO) material, NLO applications.

\section{Introduction}

The interest in future photonic devices such as frequency converters, light modulators and optical switches has led to the development of a variety of organic non-linear optical (NLO) chromophores. . $^{1-3}$

In the last few years, thiophene containing donor-acceptor substituted $\pi$ systems have been extensively investigated. ${ }^{4-19}$

These novel push-pull systems exibit enhanced second-order polarizabilities $\beta$ compared to biphenyls or stilbenes. ${ }^{14,16}$ Donor-acceptor substituted oligothiophenes represent promising candidates for NLO applications. ${ }^{1-4,13,17,20}$ 
The synthesis of donor-acceptor oligothiophenes may be achieved by several methods such as cross-coupling reactions; Stille, ${ }^{14-17,21-24}$ Suzuki, 25 or others $4,6,8,26-28$ and by procedures involving thiophene ring formations. $19,29-30$

Recently we have developed an efficient method for the synthesis of 5-amino- and 5alkoxy-2,2'-bithiophenes. ${ }^{31}$ These compounds have proved to be versatile substrates in formylation, dicyanovinylation and tricyanovinylation reactions, permitting the preparation of several new donor-acceptor substituted bithiophenes. ${ }^{32}$

As part of our continuing interest in non-linear optical material ${ }^{32-36}$ we report here the use of the readily available 5-amino- and 5-alkoxy-2,2'-bithiophenes in the Stille crosscoupling reaction with phenyl, thienyl and bithienyl bromides to obtain new donoracceptor substituted oligothiophenes.

The Stille coupling was chosen because it is one of the most versatile methods for C-C bond formation for several reasons: i) the organostannanes are readily prepared, purified and stored; ii) the Stille conditions tolerate a wide variety of functional groups (e.g. $\mathrm{CO}_{2} \mathrm{R}, \mathrm{CN}, \mathrm{OH}, \mathrm{CHO}, \mathrm{NO}_{2}$ ); iii) the reaction can be performed under mild conditions and iv) in contrast to the Suzuki reaction, the Stille coupling can be run under neutral conditions. ${ }^{37-38}$

\section{Results and discussion}

Synthesis

A series of chromophores was synthesized with either alcoxy- or $N, N$-dialkylaminodonors and formyl, nitro and dicyanovinyl acceptors across a conjugated $\pi$-bridge containing a bithiophene-benzene, terthiophene or tetrathiophene moiety.

The bithiophenes 9d, 10a-d, 11d, the terthiophenes 12b, 13-15d and the quaterthiophene 16d were synthesized by $\mathrm{Pd}\left(\mathrm{PPh}_{3}\right)_{4}$ catalyzed cross coupling reactions of (tributylstannyl)bithiophenes 8a-d with the acceptor-substituted bromo-aryl or heteroaryl compounds 1a-b, d, 2a-c and $\mathbf{6}$.

The aryl, thienyl and the bithienyl bromides used were activated by electron withdrawing substituents such as formyl, nitro and dicyanovinyl. The bromo derivatives 1-bromo-4-cyanobenzene 1a, 1-bromo-4-nitrobenzene 1b, 4-bromo-1-formylbenzene 1c, 5-bromo-2-formylthiophene $\mathbf{2 a}$ and 5-bromo-2-nitrothiophene $\mathbf{2 b}$ were commercially available. The synthesis of the other bromo derivatives was achieved by 
several methods. Knoevenagel condensation ${ }^{39}$ of the commercial available 4-bromo-1formylbenzene 1c and 5-bromo-2-formylthiophene 2a with malononitrile in refluxing ethanol gave the corresponding dicyanovinyl derivatives 4-bromo-1dicyanovinylbenzene 1d and 5-bromo-2-dicyanovinylthiophene 2c in 87 and 91\% yield respectively.

5'-Bromo-5-dicyanovinyl-2,2'-bithiophene 6 was obtained from 5-dicyanovinyl-2,2'bithiophene 5 by bromination with NBS in a solution of chloroform-acetic acid (1:1) in $85 \%$ yield. Compound 5 was obtained in 55\% yield, by Stille coupling of (tributhylstannyl)thiophene $4^{40}$ under $\mathrm{Pd}\left(\mathrm{PPh}_{3}\right)_{4}$ catalysis at $80{ }^{\circ} \mathrm{C}$ in toluene. Compound 4 was synthesized from the commercially available 2-bromothiophene 3 in quantitative yield, by lithiation, using $n$-BuLi at $0{ }^{\circ} \mathrm{C}$, followed by transmetalation with tributyltin chloride at $-78^{\circ} \mathrm{C}$ (Scheme 1).

\section{$<$ SCHEME 1 $>$}

The bromo derivatives 1d, 2c and $\mathbf{6}$ described earlier were synthesized in order to be coupled under Stille conditions with the stannane bithiophenes 8a-d.

The synthesis of bithienylstannanes 8a-d was achieved by metalation of 5-alkoxy- and 5- $N, N$-dialkylamino-2,2'-bithiophenes 7a-d, using $n$-BuLi at $0{ }^{\circ} \mathrm{C}$ followed by transmetalation with tributyltin chloride at $-78{ }^{\circ} \mathrm{C}$ (Scheme 2). The organotin compounds 8a-d were obtained in good yields (81-90\%) and were used in the Stille coupling reactions without further purification.

The Stille reactions were performed in toluene under an argon atmosphere and $\mathrm{Pd}\left(\mathrm{PPh}_{3}\right)_{4}\left(2 \mathrm{~mol} \%\right.$ ) was used as palladium catalyst at $80{ }^{\circ} \mathrm{C}$ for 8-33.5 h (Scheme 2).

\section{<SCHEME 2>}

The donor-acceptor oligothiophenes were obtained in moderate to good yields $42-65 \%$ (Table 1). Better yields were obtained when more activated aryl or thienyl bromides were used in the Stille couplings. Therefore, bithiophene 10a was synthesised in $65 \%$ yield (Table 1, entry 2) and terthiophene 15d was obtained in 55\% yield (Table 1, entry 10). 
The influence of the activation of the aryl or heteroaryl bromides on the yield of the Stille coupling is demonstrated by comparison of the yield of $9 \mathbf{d}$ (43\%) (Table 1, entry 1) with the yield of 11d (56\%) (Table 1, entry 6). A better yield was obtained for compound 11d due to the activation of the bromide 1d by the dicyanovinyl group.

\section{$<$ TABLE 1 >}

Waite 41 et al reported the study of the polarizability and hyperpolarizability of terthiophene 12b but no analytical data was described for this compound.

\section{UV-visible study of oligothiophenes}

Electronic absorption spectra of all the push-pull compounds 9-16 show an intense lowest energy charge-transfer absorption band in the UV-vis. region. The position of this band is strongly influenced by the structure of the compounds, for example by the type of $\pi$ bridge and the substitution pattern in the donor and acceptor moieties ${ }^{19}$ (Table $1)$.

The influence of the strength of the acceptor group is demonstrated by comparison of the absorption maxima of compounds 13d and 15d as the longest wavelength transition is shifted from $456.0 \mathrm{~nm}$ in piperidino- $\mathrm{T}_{3}-\mathrm{CHO}$ 13d (Table 1, entry 8 ) to $545.5 \mathrm{~nm}$ in piperidino- $\mathrm{T}_{3}-\left[\mathrm{CH}=\mathrm{C}(\mathrm{CN})_{2}\right] \mathbf{1 5 d}$ (Table 1, entry 10). The influence of the strength of the donor group is demonstrated by comparison of the absorption maxima of compounds 10a and 10c as the longest wavelength transition is shifted from $413.0 \mathrm{~nm}$ in methoxy- $\mathrm{T}_{2}-4-\mathrm{NO}_{2}-\mathrm{Ph} \mathrm{10a}$ (Table 1 , entry 2 ) to $474.5 \mathrm{~nm}$ in $N, N$-diethyl- $\mathrm{T}_{2}-4-\mathrm{NO}_{2}-$ Ph 10c (Table 1, entry 4).

In general, the stronger the donor and/or acceptor group, the smaller the energy difference between ground and excited states, and the longer the wavelength of absorption. ${ }^{14}$ According to Zyss ${ }^{1}$ the increase of the $\beta$ values characteristic of the NLO effects is accompanied by an increase of the $\lambda_{\max }$ in the UV-vis spectra.

Comparison of the electronic absorption spectra of piperidino- $\mathrm{T}_{2}-4-\mathrm{NO}_{2}-\mathrm{Ph}$ 10d (Table 1, entry 5) $\left(\lambda_{\max }=453.0 \mathrm{~nm}\right)$ with piperidino- $\mathrm{T}_{3}-\mathrm{NO}_{2}$ 14d (Table 1 , entry 9$)\left(\lambda_{\max }=\right.$ $504 \mathrm{~nm}$ ) reveals that the replacement of a benzene ring with a thiophene ring causes a dramatic red shift of the charge-transfer band. This observation clearly indicates that 
the incorporation of thiophene moieties in push-pull compounds enhances their chargetransfer properties. $4,8,13,20$

\section{Solvatochromic behavior of oligothiophenes}

Solvatochromism is easily quantified by UV-vis spectroscopy and is particularly suitable for the empirical determination of the polarity of a solvent ${ }^{42-43}$ on a molecularmicroscopic level. To evaluate the intermolecular forces between the solvents and the solute molecules we have measured absorption spectra of six oligothiophenes in 14 solvents of different solvatation character.

The maxima of the wavenumbers $v_{\max }$ for compounds 10d, 11d, 12b, 13d, 15d and 16d, as well as the corresponding wavelength $\lambda$ are listed in Table 2 and compared with the $\pi^{*}$ determined by Kamlet and Taft.

The highest energy transitions are found with nonpolar solvents such as hexane and cyclohexane. More polar solvents such as DMF resulted in lower energy transitions. This behaviour has been defined as a positive solvatochromic response (between $\Delta v=1333 \mathrm{~cm}^{-1}$ for $\mathbf{1 6 d}$ and $\Delta v=3758 \mathrm{~cm}^{-1}$ for $\mathbf{1 1 d}$ ) that is related to a greater stabilization of the excited state relative to the ground state with increasing polarity of the solvent.

Because of the pronounced solvatochromism, the good correlation with $\pi^{*}$ values for the 14 solvents investigated $(r=0.8495)$ and the long wavelength absorption in the visible range, 11d seemed to be a very appropriate solvent polarity indicating dye (Table 3). The change in dipole moment on electronic excitation was shown to be oriented parallel to the transition dipole and is moreover constant over the whole charge transfer band.

The great number of aliphatic and dipolar aprotic solvents was chosen to determine the correlation behaviour of $v_{\max }(\mathbf{1 1 d})$ and $\pi^{*}$ because specific interactions were not expected. In fact a good correlation between absorption wavenumbers of 11d and $\pi^{*}$ values $(r=0.9431)$ of the corresponding solvents was obtained (Table 2$)$.

However, as shown in Figure 1, the alcohols, aromatic and chlorinated solvents slightly deviate from this regression line. The behaviour in chlorinated and aromatic solvents, which display the lowest energy transitions is noteworthy. Similar behaviour has been observed for donor-acceptor molecules of oligothiophenes where the trend was rationalized as a consequence of an intramolecular charge transfer. ${ }^{14}$ 


\section{<TABLE 2>}

\section{$<$ TABLE 3>}

The oligothiophene derivatives 9-16 were completely characterized by $\mathrm{HRMS},{ }^{1} \mathrm{H}$ spectroscopy and by IR and UV-Vis. spectroscopy.

The study of the thermal stability, the electrochemical and the non-linear optical properties of the new oligothiophenes are under way.

\section{Experimental}

${ }^{1} \mathrm{H}$ NMR spectra were obtained on a Varian Unity Plus Spectrometer at $300 \mathrm{MHz}$ using the solvent peak as internal reference. The solvents are indicated in parenthesis before the chemical shift values ( $\delta$ relative to TMS). Mp were determined on a Gallenkamp apparatus and are uncorrected. Infrared spectra were recorded on a Perkin Elmer 1600 FTIR spectrophotometer. UV-vis spectra were recorded with a Shimadzu UV/2501/PC spectrophotometer using several solvents analytically pure (Merck). EI mass spectra EI (70 eV) and HRMS were run on a Unicam GC-MS 120. Elemental analysis was carried out on a Leco CHNS-932. Column chromatography was performed on Merck silica gel 60 (Art 9385). Light petroleum refers to solvent boiling in the range 40-60 ${ }^{\circ} \mathrm{C}$.

All reactions were carried out under an argon atmosphere in dry glassware.

The phenyl and thienyl bromides 1a-c, 2a-b and $\mathbf{3}$ were purchased from Aldrich and used as received.

The synthesis of bithiophenes $7 \mathbf{a}-\mathbf{d}$ has been described elsewhere. ${ }^{31}$

\section{General procedure for the synthesis of dicyanovinyl derivatives $1 \mathrm{~d}$ and $2 \mathrm{c}$ from the corresponding formyl compounds 1c and 2a by Knoevenagel condensation}

To a solution of malononitrile $(1.2 \mathrm{~g}, 18 \mathrm{mmol})$ and the formyl derivatives $1 \mathbf{c}$ and $2 \mathrm{a}$ $(15 \mathrm{mmol})$ in ethanol $(50 \mathrm{ml})$ was added piperidine (1 drop). The solution was heated at 
reflux for $1 \mathrm{~h}$, then cooled and the solvent was removed under reduced pressure to give the crude dicyanovinyl compounds. The resulting solids were recrystallized to give the title compounds $\mathbf{1 d}$ and $\mathbf{2 c}$.

4-Bromo-1-dicyanovinylbenzene 1d: beige solid (87\%). Mp: $160.5-161.6{ }^{\circ} \mathrm{C}$. (ether $/ n$ hexane). IR (Nujol) $v 2224(\mathrm{CN}) \mathrm{cm}^{-1} .{ }^{1} \mathrm{H}$ NMR $\left(\mathrm{CDCl}_{3}\right) \delta 7.70(\mathrm{~d}, 2 \mathrm{H}, J=8.4 \mathrm{~Hz}$, 2xAr-H), $7.73\left(\mathrm{~s}, 1 \mathrm{H}, \mathrm{CH}=\mathrm{C}(\mathrm{CN})_{2}\right), 7.78(\mathrm{~d}, 2 \mathrm{H}, J=8.4 \mathrm{~Hz}, 2 \mathrm{xAr}-H)$. Anal. Calcd for $\mathrm{C}_{10} \mathrm{H}_{5} \mathrm{BrN}_{2}$ : C, 51.52; H; 2.15; N, 12.02. Found C, 51.34; H, 2.46; N, $11.84 \%$ ).

5-Bromo-2-dicyanovinylthiophene 2c: pale orange solid (91\%). Mp: 157-158 ${ }^{\circ} \mathrm{C}$. (ether/n-hexane). UV (Acetonitrile): $\lambda_{\max } \mathrm{nm}\left(\varepsilon, / \mathrm{M}^{-1} \mathrm{~cm}^{-1}\right) 317.5,(17000)$. IR (Nujol) v 3310, $2224(\mathrm{CN}) \mathrm{cm}^{-1} .{ }^{1} \mathrm{H} \mathrm{NMR}\left(\mathrm{CDCl}_{3}\right) \delta 7.25(\mathrm{~d}, 1 \mathrm{H}, J=4.0 \mathrm{~Hz}, 4-\mathrm{H}), 7.51(\mathrm{~d}, 1 \mathrm{H}$, $J=4.0 \mathrm{~Hz}, 3-\mathrm{H}), 7.75$ (s, $\left.1 \mathrm{H}, \mathrm{CH}=\mathrm{C}(\mathrm{CN})_{2}\right)$. MS (EI) m/z (\%): $240\left(\mathrm{M}^{+}{ }^{81} \mathrm{Br}, 98\right), 238$ $\left(\mathrm{M}^{+}{ }^{79} \mathrm{Br}, 100\right), 189$ (10), 187 (10), 159 (51). HRMS: $m / z$ (EI) for $\mathrm{C}_{8} \mathrm{H}_{3}{ }^{81} \mathrm{BrN}_{2} \mathrm{~S}$; calcd 239.9180; found: 239.9180. Anal. Calcd for $\mathrm{C}_{8} \mathrm{H}_{3} \mathrm{BrN}_{2} \mathrm{~S}$ : C, 40.17; $\mathrm{H} ; 1.26 ; \mathrm{N}, 11.72$; S, 13.39. Found C, 40.23; H, 1.49; N, 11.44\%).

\section{Synthesis of 5'-bromo-5-dicyanovinyl-2,2'-bithiophene 6}

\section{i) Synthesis of 2-(tri-n-butylstannyl)thiophene $4^{40}$}

Under argon a solution of $n$-BuLi in hexanes $(2.5 \mathrm{ml}, 6.14 \mathrm{mmol}, 2.5 \mathrm{M})$ was dropped into a stirred solution of $3(3.07 \mathrm{mmol})$ in dry ether at $0{ }^{\circ} \mathrm{C}$. After $1 \mathrm{~h}$ the mixture was cooled to $-78{ }^{\circ} \mathrm{C}$ and a solution of tributyltin chloride $(1 \mathrm{~g} / 0.83 \mathrm{ml}, 3.07 \mathrm{mmol})$ in dry ether was slowly added and the mixture was stirred overnight. The mixture was then added to water $(50 \mathrm{ml})$. The aqueous layer was extracted with ether $(3 \times 30 \mathrm{ml})$. The combined organic layers were dried with magnesium sulfate, and the solvent was removed in vacuo to give the title product 4 as a pale brown oil in quantitative yield. ${ }^{1} \mathrm{H}$ NMR $\left(\mathrm{CDCl}_{3}\right) \delta$ 0.80-1.00 (m, $\left.\left.15 \mathrm{H}, 3 \mathrm{x}\left(\mathrm{CH}_{2}\right)_{2} \mathrm{CH}_{2} \mathrm{CH}_{3}\right)\right), 1.10-1.50(\mathrm{~m}, 12 \mathrm{H}$, $\left.3 \times\left(\mathrm{CH}_{2}\right)_{2} \mathrm{CH}_{2} \mathrm{CH}_{3}\right)$ ), 7.20 (dd, $1 \mathrm{H}, J=3.3$ and $\left.1.0 \mathrm{~Hz}, 3-\mathrm{H}\right), 7.25-7.29(\mathrm{~m}, 1 \mathrm{H}, 4-\mathrm{H})$, $7.66(\mathrm{dd}, 1 \mathrm{H}, J=4.7$ and $1.0 \mathrm{~Hz}, 5-\mathrm{H})$. Product 4 was used in the Stille coupling without further purification. 


\section{ii) Synthesis of 5-dicyanovinyl-2,2'-bithiophene 5}

A degassed solution of the 5-bromo-2-dicyanovinylthiophene 2c (0.66 g, $2.8 \mathrm{mmol})$, the thienylstananne $4(3.07 \mathrm{mmol})$ and $\mathrm{Pd}\left(\mathrm{PPh}_{3}\right)_{4}(0.056 \mathrm{mmol})$ in toluene $(5 \mathrm{ml})$ was heated at $80{ }^{\circ} \mathrm{C}$ under argon. After $24 \mathrm{~h}$ the reaction mixture was cooled to room temperature, filtered and washed with a cold mixture of ether/petrol to give the pure 5dicyanovinyl-2,2'-bithiophene 5 as a pale orange solid. The organic solution obtained from the filtration was washed with a saturated solution of KF $(3 \times 50 \mathrm{ml})$, water $(3 \times 50$ $\mathrm{ml})$ and a saturated solution of $\mathrm{NaCl}(100 \mathrm{ml})$. The resulting organic layer were dried with magnesium sulfate, and the solvent was removed in vacuo to give a brown oil. Overall yield: $55 \%$. Recrystallization from $n$-hexane gave the pure 5-dicyanovinyl2,2"'-bithiophene 5 as a pale orange solid mp: 166.5-168.5 ${ }^{\circ} \mathrm{C}$. IR (Nujol) $v 2218$ (CN) $\mathrm{cm}^{-1} .{ }^{1} \mathrm{H}$ NMR $\left(\mathrm{CDCl}_{3}\right) \delta$ 7.18-7.22 (m, $1 \mathrm{H}, 4$ '-H), 7.62 (d, $\left.1 \mathrm{H}, J=4.5 \mathrm{~Hz}, 3-\mathrm{H}\right), 7.67$ (dd, $1 \mathrm{H}, J=3.8$ and $\left.1.0 \mathrm{~Hz}, 3^{\prime}-\mathrm{H}\right), 7.78$ (dd, $1 \mathrm{H}, J=5.0$ and $\left.1.0 \mathrm{~Hz}, 5^{\prime}-\mathrm{H}\right), 7.89$ (d, $1 \mathrm{H}$, $J=4.5 \mathrm{~Hz}, 3-\mathrm{H}), 8.64\left(\mathrm{~s}, 1 \mathrm{H}, \mathrm{CH}=\mathrm{C}(\mathrm{CN})_{2}\right)$.

\section{iii) Synthesis of 5'-bromo-5-dicyanovinyl-2,2'-bithiophene 6}

To a stirred solution of 5-dicyanovinyl-2,2'-bithiophene $5(0.1 \mathrm{~g}, 0.41 \mathrm{mmol})$ in a 1:1 $(\mathrm{v} / \mathrm{v})$ solution of chloroform-acetic acid $(12 \mathrm{ml})$ was added NBS $(0.073 \mathrm{~g}, 0.41 \mathrm{mmol})$ at $\mathrm{rt}$. After $24 \mathrm{~h}$ the reaction mixture was washed with water $(30 \mathrm{ml})$. The organic layer was dried with magnesium sulfate, and the solvent was removed in vacuo to give the pure bithiophene 6 as a orange brownish solid (85\%). Recrystallization from $n$-hexane gave the title compound as a pale orange solid mp: 193-195 ${ }^{\circ} \mathrm{C}$. UV (Acetonitrile): $\lambda_{\max } \mathrm{nm}\left(\varepsilon, / \mathrm{M}^{-1} \mathrm{~cm}^{-1}\right) 421.0$ (21290), 308.0 (240). IR (Nujol) $v 2222(\mathrm{CN}) \mathrm{cm}^{-1}$. ${ }^{1} \mathrm{H}$ NMR (DMSO) $\delta 7.35$ (d, $1 \mathrm{H}, J=4.2 \mathrm{~Hz}, 3$ '-H), 7.53 (d, $1 \mathrm{H}, J=4.2 \mathrm{~Hz}, 4$ '-H), 7.61 (d, $1 \mathrm{H}, J=4.5 \mathrm{~Hz}, 3-\mathrm{H}), 7.88$ (d, $1 \mathrm{H}, J=4.5 \mathrm{~Hz}, 4-\mathrm{H}), 8.65$ (s, $\left.1 \mathrm{H}, \mathrm{CH}=\mathrm{C}(\mathrm{CN})_{2}\right)$. MS (EI) $\mathrm{m} / \mathrm{z} \quad(\%): 322\left(\mathrm{M}^{+}{ }^{81} \mathrm{Br}, 99\right), 320\left(\mathrm{M}^{+79} \mathrm{Br}, 100\right)$. HRMS: $m / z$ (EI) for $\mathrm{C}_{12} \mathrm{H}_{5}{ }^{81} \mathrm{BrN}_{2} \mathrm{~S}_{2}$; calcd 321.9057; found: 321.9058 . 
General procedure for the synthesis of 2-alkoxy- and 2-amino-substituted 5-(tri-nbutylstannyl)-2,2'-bithiophenes 8a-d

Under Ar a solution of $n$-BuLi in hexanes $(1.3 \mathrm{ml}, 3.21 \mathrm{mmol}, 2.5 \mathrm{M})$ was dropped into a stirred solution of $7(2.7 \mathrm{mmol})$ in dry ether at $0{ }^{\circ} \mathrm{C}$. After $1 \mathrm{~h}$ the mixture was cooled to $-78{ }^{\circ} \mathrm{C}$ and a solution of tri-n-butylchlorostannane $(2.7 \mathrm{mmol})$ in dry ether was slowly added and the mixture was stirred overnight. The mixture was then added to water $(50 \mathrm{ml})$. The aqueous layer was extracted with ether $(3 \times 30 \mathrm{ml})$. The combined organic layers were dried with magnesium sulfate, and the solvent was removed in vacuo to give product 8. Derivatives 8a-d were used in the Stille couplings without further purification.

5-Methoxy-5'-(tri-n-butylstannyl)-2,2'-bithiophene 8a: green oil (85\%). ${ }^{1} \mathrm{H}$ NMR $\left.\left(\mathrm{CDCl}_{3}\right) \quad \delta 0.80-1.00\left(\mathrm{~m}, \quad 15 \mathrm{H}, \quad 3 \times\left(\mathrm{CH}_{2}\right)_{2} \mathrm{CH}_{2} \mathrm{CH}_{3}\right)\right), \quad 1.20-1.40 \quad(\mathrm{~m}, 12 \mathrm{H}$, $\left.\left.3 \times\left(\mathrm{CH}_{2}\right)_{2} \mathrm{CH}_{2} \mathrm{CH}_{3}\right)\right), 3.90\left(\mathrm{~s}, 3 \mathrm{H}, \mathrm{OCH}_{3}\right), 5.80$ (d, $\left.1 \mathrm{H}, J=3.9 \mathrm{~Hz}, 4-\mathrm{H}\right), 6.87$ (d, $1 \mathrm{H}$, $J=3.9 \mathrm{~Hz}, 3-\mathrm{H}), 7.00$ (d, $1 \mathrm{H}, J=3.6 \mathrm{~Hz}, 3$ '-H), 7.07 (d, $1 \mathrm{H}, J=3.6 \mathrm{~Hz}, 4^{\prime}-\mathrm{H}$ ).

5- $N, N$-Dimethylamino-5' -(tri-n-butylstannyl)-2,2'-bithiophene 8b: orange oil $(90 \%) .{ }^{1} \mathrm{H}$ NMR $\left.\left(\mathrm{CDCl}_{3}\right) \delta 0.80-1.00\left(\mathrm{~m}, 15 \mathrm{H}, 3 \mathrm{x}\left(\mathrm{CH}_{2}\right)_{2} \mathrm{CH}_{2} \mathrm{CH}_{3}\right)\right), 1.10-1.45(\mathrm{~m}, 12 \mathrm{H}$, $\left.\left.3 \mathrm{x}\left(\mathrm{CH}_{2}\right)_{2} \mathrm{CH}_{2} \mathrm{CH}_{3}\right)\right), 2.93\left(6 \mathrm{H}, \mathrm{s}, \mathrm{N}\left(\mathrm{CH}_{3}\right)_{2}\right), 5.80$ (d, $\left.1 \mathrm{H}, J=3.7 \mathrm{~Hz}, 4-\mathrm{H}\right), 6.87$ (d, 1 H, J=3.7 Hz, 3-H), 7.00 (d, 1 H, J=3.5 Hz, 4'-H) 7.07 (d, 1 H, J=3.5 Hz, 3'-H).

5- $N, N$-Diethylamino-5'-(tri-n-butylstannyl)-2,2'-bithiophene $\quad 8 \mathrm{c}$ : pale brown oil (90\%). ${ }^{1} \mathrm{H}$ NMR $\left(\mathrm{CDCl}_{3}\right) \delta$ 0.80-1.00 (m, $\left.\left.15 \mathrm{H}, 3 \mathrm{x}\left(\mathrm{CH}_{2}\right)_{2} \mathrm{CH}_{2} \mathrm{CH}_{3}\right)\right), 1.10-1.45(\mathrm{~m}, 12$ $\mathrm{H}, 3 \mathrm{x}\left(\mathrm{CH}_{2}\right)_{2} \mathrm{CH}_{2} \mathrm{CH}_{3}$ )), 1.20-1.30 (overlapped t, $6 \mathrm{H}, 2 \mathrm{xCH}_{2} \mathrm{CH}_{3}$ ), 3.25-3.35 (q, $4 \mathrm{H}$, $\left.J=6.0 \mathrm{~Hz}, 2 \mathrm{xCH}_{2} \mathrm{CH}_{3}\right), 5.78$ (d, $\left.1 \mathrm{H}, J=3.9 \mathrm{~Hz}, 4-\mathrm{H}\right), 6.86$ (d, $\left.1 \mathrm{H}, J=3.9 \mathrm{~Hz}, 3-\mathrm{H}\right), 7.00$ (d, $\left.1 \mathrm{H}, J=3.6 \mathrm{~Hz}, 4^{\prime}-\mathrm{H}\right) 7.05$ (d, $\left.1 \mathrm{H}, J=3.6 \mathrm{~Hz}, 3^{\prime}-\mathrm{H}\right)$.

5-Piperidino-5'-(tri-n-butylstannyl)-2,2'-bithiophene 8d: pale brown oil $(81 \%) .{ }^{1} \mathrm{H}$ NMR $\left.\left(\mathrm{CDCl}_{3}\right) \delta 0.80-1.00\left(\mathrm{~m}, 15 \mathrm{H}, 3 \mathrm{x}\left(\mathrm{CH}_{2}\right)_{2} \mathrm{CH}_{2} \mathrm{CH}_{3}\right)\right), 1.20-1.40(\mathrm{~m}, 12 \mathrm{H}$, $\left.\left.3 \times\left(\mathrm{CH}_{2}\right)_{2} \mathrm{CH}_{2} \mathrm{CH}_{3}\right)\right), 1.50-1.80\left(\mathrm{~m}, 6 \mathrm{H}, 3 \mathrm{xCH}_{2}\right), 3.10-3.20\left(\mathrm{~m}, 4 \mathrm{H}, 2 \mathrm{xNCH}_{2}\right), 5.98$ (d, $1 \mathrm{H}, J=3.9 \mathrm{~Hz}, 4-\mathrm{H}), 6.86$ (d, $1 \mathrm{H}, J=3.9 \mathrm{~Hz}, 3-\mathrm{H}), 7.00$ (d, $1 \mathrm{H}, J=3.6 \mathrm{~Hz}, 4$ '-H) 7.08 (d, $\left.1 \mathrm{H}, J=3.6 \mathrm{~Hz}, 3^{\prime}-\mathrm{H}\right)$. 
General procedure for palladium-catalyzed cross-couplings of aryl 1a-b, 1d and heteroaryl bromides 2a-c and 6 with stannylbithiophene derivatives 8a-d

To a degassed solution of aryl 1a-b and 1d, thienyl 2a-c or bithienyl $\mathbf{6}$ bromides $(0.5$ $\mathrm{mmol})$, and bithienylstanannes 8a-d $(0.55 \mathrm{mmol})$ in toluene $(5 \mathrm{ml})$ was added $\mathrm{Pd}\left(\mathrm{PPh}_{3}\right)_{4}(0.01 \mathrm{mmol})$. The mixture was heated at $80{ }^{\circ} \mathrm{C}$ under argon. After the given reactions times (TLC control, Table 1) the reaction mixture was cooled to room temperature and then filtered and washed with cold toluene to give the pure oligothiophenes 9d, 10a-d, 11d, 12b and 13d-16d. The isolated solids were recrystallized. The organic solution obtained from the filtration was washed with a saturated solution of $\mathrm{KF}(3 \times 30 \mathrm{ml})$, water $(3 \times 30 \mathrm{ml})$ and a saturated solution of $\mathrm{NaCl}$ $(50 \mathrm{ml})$. The resulting organic layers were dried with magnesium sulfate, and the solvent was removed in vacuo to give oils which by ${ }^{1} \mathrm{NMR}$ reveal to be the stannanes derivatives used in excess.

5'-(4''-Cyanophenyl)-5-piperidino-2,2'-bithiophene 9d: orange solid (43\%). Mp: 227$229{ }^{\circ} \mathrm{C}$ (ether). UV (EtOH): $\lambda_{\max } \mathrm{nm}\left(\varepsilon, / \mathrm{M}^{-1} \mathrm{~cm}^{-1}\right) 420.0$ (18660), 297.0 (7450), $255.0 \mathrm{~s}$ (8430), 239.0 (11130), $215.0 \mathrm{~s}$ (13340). IR (Nujol) $v 2219(\mathrm{CN}) \mathrm{cm}^{-1} .{ }^{1} \mathrm{H} \mathrm{NMR}$ $\left(\mathrm{CDCl}_{3}\right) \delta 1.50-1.80\left(\mathrm{~m}, 6 \mathrm{H}, 3 \mathrm{xCH}_{2}\right), 3.10-3.20\left(\mathrm{~m}, 4 \mathrm{H}, 2 \mathrm{xNCH}_{2}\right), 6.00(\mathrm{~d}, 1 \mathrm{H}, J=3.9$ Hz, 4-H), 6.92-6.98 (m, 2 H, 3 and 3'-H), 7.30 (d, 1 H, J=4.2 Hz, 4'-H), 7,63 (br s, 4 H, 4xAr-H). MS (EI) m/z (\%): $350\left(\mathrm{M}^{+}, 100\right)$. HRMS: $\mathrm{m} / \mathrm{z}(\mathrm{EI})$ for $\mathrm{C}_{20} \mathrm{H}_{18} \mathrm{~N}_{2} \mathrm{~S}_{2}$; calcd 350.0911; found: 350.0916 .

5-Methoxy-5'-(4''-nitrophenyl)-2,2'-bithiophene 10a: orange solid (65\%). Mp: 169$171{ }^{\circ} \mathrm{C}$ (ether). UV (EtOH): $\lambda_{\max } \mathrm{nm}\left(\varepsilon, / \mathrm{M}^{-1} \mathrm{~cm}^{-1}\right) 413.0$ (25750), 289.0 (8680), 252.0 (1450), $213.0 \mathrm{~s}$ (1810). IR (Nujol) v 1593, 1531, 1505, 1351, 1200, 1158, 1111, 1048, 846, 800, 772, 749, 721, $666 \mathrm{~cm}^{-1} .{ }^{1} \mathrm{H} \mathrm{NMR}\left(\mathrm{CDCl}_{3}\right) \delta 3.94\left(\mathrm{~s}, 3 \mathrm{H}, \mathrm{OCH}_{3}\right)$, 6.18 (d, $1 \mathrm{H}, J=3.9 \mathrm{~Hz}, 4-\mathrm{H}), 6.91$ (d, $1 \mathrm{H}, J=3.9 \mathrm{~Hz}, 3-\mathrm{H}), 7.03$ (d, $1 \mathrm{H}, J=3.9 \mathrm{~Hz}, 3$ 'H), 7,37 (d, $1 \mathrm{H}, J=3.9 \mathrm{~Hz}, 4$ '-H), 7.70 (d, $2 \mathrm{H}, J=9.0 \mathrm{~Hz}, 2$ ' ' and 6' '-H), 8.23 (d, $2 \mathrm{H}$, $J=9.0 \mathrm{~Hz}, 3$ ' , and 5'-H). MS (EI) m/z (\%): $317\left(\mathrm{M}^{+}, 44\right)$. Anal. Calcd for $\mathrm{C}_{15} \mathrm{H}_{11} \mathrm{NO}_{3} \mathrm{~S}_{2}$ : C, 56.76; H, 3.47; N, 4.41; S, 20.20. Found: C, 56.51; H, 3.52; N, 4.44; S, 19.80. HRMS: $\mathrm{m} / \mathrm{z}(\mathrm{EI})$ for $\mathrm{C}_{15} \mathrm{H}_{11} \mathrm{NO}_{3} \mathrm{~S}_{2}$; calcd 317.0180; found: 317.0174 . 
5-N,N-Dimethyl-5'-(4' '-nitrophenyl)-2,2'-bithiophene 10b: orange solid (42\%). Mp: 243-245 ${ }^{\circ} \mathrm{C}$ (ethanol). UV (EtOH): $\lambda_{\max } \mathrm{nm}\left(\varepsilon, / \mathrm{M}^{-1} \mathrm{~cm}^{-1}\right) 461.0$ (10050), 322.0 (4650), 264.0 (5140), 211.0 s (8540). IR (Nujol) v1592, 1563, 1534, 1504, 1450, 1425, $1331,1278,110,1056,919,848,795,748,688,666 \mathrm{~cm}^{-1} .{ }^{1} \mathrm{H} \mathrm{NMR}\left(\mathrm{CDCl}_{3}\right) \delta 2.98(\mathrm{~s}$, $\left.6 \mathrm{H}, 2 \mathrm{xCH}_{3}\right), 5.81(\mathrm{~d}, 1 \mathrm{H}, J=3.9 \mathrm{~Hz}, 4-\mathrm{H}), 6.94$ (d, $\left.1 \mathrm{H}, J=3.9 \mathrm{~Hz}, 3-\mathrm{H}\right), 6.96$ (d, $1 \mathrm{H}$, $J=3.9 \mathrm{~Hz}, 3$ '-H), 7,35 (d, $1 \mathrm{H}, J=3.9 \mathrm{~Hz}, 4$ '-H), 7.65 (d, $2 \mathrm{H}, J=8.9 \mathrm{~Hz}, 2$ '' and 6' '-H), $8.20\left(\mathrm{~d}, 2 \mathrm{H}, \mathrm{J}=8.9 \mathrm{~Hz}, 3^{\prime}\right.$ ' and 5' '-H). MS (EI) m/z (\%): $330\left(\mathrm{M}^{+}, 100\right)$. HRMS: $\mathrm{m} / \mathrm{z}$ (EI) for $\mathrm{C}_{16} \mathrm{H}_{14} \mathrm{~N}_{2} \mathrm{O}_{2} \mathrm{~S}_{2}$; calcd 330.0497; found: 330.0505 .

5-N,N-Diethyl-5'-(4''-nitrophenyl)-2,2'-bithiophene 10c: dark red solid (44\%). Mp: 181-183 ${ }^{\circ} \mathrm{C}$ (ethanol). UV (EtOH): $\lambda_{\max } \mathrm{nm}\left(\varepsilon, / \mathrm{M}^{-1} \mathrm{~cm}^{-1}\right) 474.5$ (16800), 360.0 (9630), 265.0 (7140). IR (Nujol) v 1591, 1504, 1332, 1280, 1183, 1108, 1057, 847, 791, 750, 722, $666 \mathrm{~cm}^{-1} .{ }^{1} \mathrm{H} \mathrm{NMR}\left(\mathrm{CDCl}_{3}\right) \delta 1.24\left(\mathrm{t}, 6 \mathrm{H}, J=7.0 \mathrm{~Hz}, 2 \mathrm{xCH}_{2} \mathrm{CH}_{3}\right), 3.34(\mathrm{q}, 4$ $\left.\mathrm{H}, J=7.0 \mathrm{~Hz}, 2 \mathrm{xCH}_{2} \mathrm{CH}_{3}\right), 5.79$ (d, $\left.1 \mathrm{H}, J=3.9 \mathrm{~Hz}, 4-\mathrm{H}\right), 6.92$ (d, $\left.1 \mathrm{H}, J=3.9 \mathrm{~Hz}, 3-\mathrm{H}\right)$, 6.98 (d, 1 H, J=3.9 Hz, 3'-H), 7,35 (d, 1 H, J=3.9 Hz, 4'-H), 7.66 (d, 2 H, J=9.0 Hz, 2', and 6"'-H), 8.21 (d, $2 \mathrm{H}, \mathrm{J}=9.0 \mathrm{~Hz}, 3$ "' and 5' -H). MS (EI) m/z (\%): $358\left(\mathrm{M}^{+}, 100\right)$. HRMS: $m / z$ (EI) for $\mathrm{C}_{18} \mathrm{H}_{18} \mathrm{~N}_{2} \mathrm{O}_{2} \mathrm{~S}_{2}$; calcd 358.0810; found: 358.0810 .

5-Piperidino-5'-(4' '-nitrophenyl)-2,2'-bithiophene 10d: brown solid (53\%). Mp: 238$240{ }^{\circ} \mathrm{C}$ (ethanol). UV (EtOH): $\lambda \max \mathrm{nm}\left(\varepsilon, / \mathrm{M}^{-1} \mathrm{~cm}^{-1}\right) 453.0$ (10000), 322.0 (4720), 316.0 (4710), 266.0 (4700), $213.0 \mathrm{~s}$ (8611). IR (Nujol) v 1592, 1504, 1493, 1329, $1275,1247,1117,1066,843,796,686,666 \mathrm{~cm}^{-1} .{ }^{1} \mathrm{H}$ NMR $\left(\mathrm{CDCl}_{3}\right) \delta 1.50-1.80(\mathrm{~m}, 6$ $\mathrm{H}, 3 \mathrm{xCH}_{2}$ ), 3.16-3.20 (m, $4 \mathrm{H}, 2 \mathrm{xNCH}_{2}$ ), 5.99 (d, $\left.1 \mathrm{H}, J=3.9 \mathrm{~Hz}, 4-\mathrm{H}\right), 6.95-6.98$ (m, 2 H, 3 and 3'-H), 7.35 (d, 1 H, J=3.9 Hz, 4'-H), 7.66 (d, 2 H, J=8.9 Hz, 2', and 6' '-H), $8.21\left(\mathrm{~d}, 2 \mathrm{H}, \mathrm{J}=8.9 \mathrm{~Hz}, 3^{\prime}\right.$ ' and 5' '-H). MS (EI) m/z (\%): $370\left(\mathrm{M}^{+}, 100\right)$. HRMS: $\mathrm{m} / \mathrm{z}$ (EI) for $\mathrm{C}_{19} \mathrm{H}_{18} \mathrm{~N}_{2} \mathrm{O}_{2} \mathrm{~S}_{2}$; calcd 370.0810; found: 370.0814 .

5'-(4' '-Dicyanovinylphenyl)-5-piperidino-2,2'-bithiophene 11d: green solid with metal luster (56\%). Mp: 232-233 ${ }^{\circ} \mathrm{C}$. UV (Ethanol): $\lambda_{\max } \mathrm{nm}\left(\varepsilon, / \mathrm{M}^{-1} \mathrm{~cm}^{-1}\right) 468.0$ (21400), 360.5 (13386). IR (Nujol) $v 2223(\mathrm{CN}) . \mathrm{cm}^{-1} .{ }^{1} \mathrm{H}$ NMR (DMSO-d 6 ) $\delta 1.45-1.70$ (m, 6 $\mathrm{H}, 3 \mathrm{xCH}_{2}$ ), 3.10-3.20 (m, $\left.4 \mathrm{H}, 2 \mathrm{xNCH}_{2}\right), 6.11$ (d, $\left.1 \mathrm{H}, J=4.5 \mathrm{~Hz}, 4-\mathrm{H}\right), 7.08-7.14$ (m, 2 H, 3 and 3'-H), 7.69 (d, $1 \mathrm{H}, J=3.9 \mathrm{~Hz}, 4$ '-H), 7,90 (d, $2 \mathrm{H}, J=8.4 \mathrm{~Hz}, 2$ '' and 6' '-H), 
$7.96\left(\mathrm{~d}, 2 \mathrm{H}, \mathrm{J}=8.4 \mathrm{~Hz}, 3\right.$ " and 5"--H), 8,44 (s, $\left.1 \mathrm{H}, \mathrm{CH}=\mathrm{C}(\mathrm{CN})_{2}\right)$. MS (EI) $\mathrm{m} / \mathrm{z}(\%)$ : $401\left(\mathrm{M}^{+}, 100\right)$. HRMS: $\mathrm{m} / \mathrm{z}$ (EI) for $\mathrm{C}_{23} \mathrm{H}_{19} \mathrm{~N}_{3} \mathrm{~S}_{2}$; calcd 401,1020; found: 401,1022.

5"'-Formyl-5-N,N-dimethyl-2,2':5'2"'-terthiophene 12b: brown solid (46\%). Mp: 186$188^{\circ} \mathrm{C}$ (ethanol) [lit. ${ }^{41}$ (mp not quoted)]. UV (EtOH): $\lambda \max \mathrm{nm}\left(\varepsilon, / \mathrm{M}^{-1} \mathrm{~cm}^{-1}\right) 465.5$ (22690), 342.0 (9300), 258.0 (12010), $213.0 \mathrm{~s}$ (14100). IR (Nujol) $v 1650$ (CHO) $\mathrm{cm}^{-1}$. ${ }^{1} \mathrm{H}$ NMR $\delta 2.98$ (s, $\left.6 \mathrm{H}, 2 \mathrm{xCH}_{3}\right), 5.80$ (d, $\left.1 \mathrm{H}, J=4.4 \mathrm{~Hz}, 4-\mathrm{H}\right), 6.87$ (d, $1 \mathrm{H}, J=4.4 \mathrm{~Hz}$, 4' or 3'-H), 6.96 (d, 1 H, J=3.9 Hz, 3-H), 7,17 (d, 1 H, J=4.4 Hz, 3''-H), 7.22 (d, 1 H, $J=4.4 \mathrm{~Hz}, 3$ ' or 4'-H), 7.65 (d, $1 \mathrm{H}, J=4.4 \mathrm{~Hz}, 4$ ''-H), 9.84 (s, $1 \mathrm{H}, \mathrm{CHO}$ ). MS (EI) m/z (\%): $319\left(\mathrm{M}^{+}, 100\right)$. HRMS: $\mathrm{m} / \mathrm{z}$ (EI) for $\mathrm{C}_{15} \mathrm{H}_{13} \mathrm{NOS}_{3}$; calcd 319.0159; found: 319.0156 .

5"'-Formyl-5-piperidino-2,2':5'2"-terthiophene 13d: brown solid (51\%). Mp: 178$180{ }^{\circ} \mathrm{C}$ (ether) UV (EtOH): $\lambda_{\max } \mathrm{nm}\left(\varepsilon, / \mathrm{M}^{-1} \mathrm{~cm}^{-1}\right) 456.0$ (15260), 332.0 (6000), 257.0 (7570). IR (Nujol) $v 1645(\mathrm{CHO}) \mathrm{cm}^{-1} .{ }^{1} \mathrm{H}$ NMR $\left(\right.$ DMSO-d $\left._{6}\right) \delta$ 1.50-1.70 (m, $6 \mathrm{H}$, $3 \mathrm{xCH}_{2}$ ), 3.05-3.15 (m, $\left.4 \mathrm{H}, 2 \mathrm{xNCH}_{2}\right) 6.10$ (d, $\left.1 \mathrm{H}, J=4.2 \mathrm{~Hz}, 4-\mathrm{H}\right), 7.05$ (d, $1 \mathrm{H}, J=3.9$ Hz, 4'-H), 7.09 (d, 1 H, J=4.2 Hz, 3-H), 7.45 (d, 1 H, J=4.2 Hz, 3''-H), 7.48 (d, 1 H, $J=3.9 \mathrm{~Hz}, 3$ '-H), 7.96 (d, $1 \mathrm{H}, J=4.2 \mathrm{~Hz}, 4$ ''-H), 9.84 (s, $1 \mathrm{H}, \mathrm{CHO}$ ). MS (EI) $\mathrm{m} / \mathrm{z}(\%)$ : $359\left(\mathrm{M}^{+}, 100\right)$. HRMS: $\mathrm{m} / \mathrm{z}(\mathrm{EI})$ for $\mathrm{C}_{18} \mathrm{H}_{17} \mathrm{NOS}_{3}$; calcd 359.0472; found: 359.0482 .

5"'-Nitro-5-piperidino-2,2':5'2"'-terthiophene 14d: dark red solid (53\%). Mp: 215$217{ }^{\circ} \mathrm{C}$ (ether) UV (EtOH): $\lambda_{\max } \mathrm{nm}\left(\varepsilon, / \mathrm{M}^{-1} \mathrm{~cm}^{-1}\right) 504.0$ (10100), 355.0 (2510), 219.0 (4350). IR (Nujol) v 1559, 1509, 1482, 1325, 1274, 1244, 1120, 1074, 1035, 852, 793, 759, 730, $666 \mathrm{~cm}^{-1} .{ }^{1} \mathrm{H}$ NMR (DMSO-d 6$) \delta 1.50-1.80\left(\mathrm{~m}, 6 \mathrm{H}, 3 \mathrm{xCH}_{2}\right), 3.17-3.22(\mathrm{~m}$, $4 \mathrm{H}, 2 \mathrm{xNCH}_{2}$ ), 5.99 (d, $\left.1 \mathrm{H}, J=3.9 \mathrm{~Hz}, 4-\mathrm{H}\right), 6.89$ (d, $1 \mathrm{H}, J=3.6 \mathrm{~Hz}, 4^{\prime}$ or 3'-H), 6.97 (d, $1 \mathrm{H}, J=3.9 \mathrm{~Hz}, 3-\mathrm{H}), 7,00$ (d, $1 \mathrm{H}, J=4.5 \mathrm{~Hz}, 3$ ''-H), 7.24 (d, $1 \mathrm{H}, J=3.6 \mathrm{~Hz}, 3$ ' or 4'H), 7.83 (d, $1 \mathrm{H}, J=4.5 \mathrm{~Hz}, 4$ "'). MS (EI) $\mathrm{m} / \mathrm{z}(\%): 376\left(\mathrm{M}^{+}, 100\right)$. HRMS: $\mathrm{m} / \mathrm{z}$ (EI) for $\mathrm{C}_{17} \mathrm{H}_{16} \mathrm{~N}_{2} \mathrm{O}_{2} \mathrm{~S}_{3}$; calcd 376.0374; found: 376.0363 .

5"'-Dicyanovinyl-5-piperidino-2,2':5',2"-terthiophene 15d: dark purple solid (55\%) Mp: 185-187 ${ }^{\circ} \mathrm{C} . \mathrm{UV}$ (EtOH): $\lambda_{\max } \mathrm{nm}\left(\varepsilon, / \mathrm{M}^{-1} \mathrm{~cm}^{-1}\right)$ (Ethanol) 545.5 (23770), 377.0 (10992). IR (Nujol) $v 2218(\mathrm{CN}) \mathrm{cm}^{-1} .{ }^{1} \mathrm{H}$ NMR (DMSO-d 6$)$ 1.50-1.70 (m, $6 \mathrm{H}$, $\left.3 \mathrm{xCH}_{2}\right), 3.10-3.20\left(\mathrm{~m}, 4 \mathrm{H}, 2 \mathrm{xNCH}_{2}\right), 6.13(\mathrm{~d}, 1 \mathrm{H}, J=4.2 \mathrm{~Hz}, 4-\mathrm{H}), 7.09$ (d, $1 \mathrm{H}$, 
$J=3.9 \mathrm{~Hz}, 4^{\prime}$ or 3'-H), 7.17 (d, $\left.1 \mathrm{H}, J=4.2 \mathrm{~Hz}, 3-\mathrm{H}\right), 7.55$ (d, $1 \mathrm{H}, J=4.2 \mathrm{~Hz}, 3$ ' '-H), 7.59 (d, $1 \mathrm{H}, J=3.9 \mathrm{~Hz}, 3$ ' or 4'-H), 7.86 (d, $1 \mathrm{H}, J=4.2 \mathrm{~Hz}, 4$ '’-H), 8,57 (s, $1 \mathrm{H}$, $\left.\mathrm{CH}=\mathrm{C}(\mathrm{CN})_{2}\right)$. MS (EI) $\mathrm{m} / \mathrm{z}(\%)$ : $407\left(\mathrm{M}^{+}, 100\right)$. HRMS: $m / z(\mathrm{EI})$ for $\mathrm{C}_{21} \mathrm{H}_{17} \mathrm{~N}_{3} \mathrm{~S}_{3}$; calcd 407.0585; found: 407.0594 .

5,"'Dicyanovinyl-5-piperidino-2,2':5',2"':5',2,"'-tetrathiophene 16d: dark blue solid (45\%). Mp: $>230,0{ }^{\circ} \mathrm{C}$ (with decomposition). $\lambda_{\max } \mathrm{nm}\left(\varepsilon, / \mathrm{M}^{-1} \mathrm{~cm}^{-1}\right)$ (Ethanol) 510.5 (12000). IR (Nujol) $v 2218(\mathrm{CN}) \mathrm{cm}^{-1}$. ${ }^{1} \mathrm{H}$ NMR (DMSO-d $)$ 1.42-1.70 (m, $6 \mathrm{H}$, $\left.3 \mathrm{xCH}_{2}\right), 3.10-3.20\left(\mathrm{~m}, 4 \mathrm{H}, 2 \mathrm{xNCH}_{2}\right), 6.10$ ( d, $\left.1 \mathrm{H}, J=4.2 \mathrm{~Hz}, 4-\mathrm{H}\right), 7.05$ (d, $1 \mathrm{H}$, $J=4.2 \mathrm{~Hz}, 4$ ' or 3'-H), 7.16 (d, $1 \mathrm{H}, J=4.2 \mathrm{~Hz}, 3-\mathrm{H}), 7.34-7.38$ (m, $2 \mathrm{H}, 3$ ' or 4'-H and 3' '-H or 4' '-H), 7.64 (d, 1 H, J=4.2 Hz, 3','-H), 7.67 (d, 1 H, J=4.2 Hz, 4' 'or 3' '-H), 7.89 (d, $1 \mathrm{H}, J=4.2 \mathrm{~Hz}, 4$ ', '-H), 8.62 (s, 1H, CH=C(CN)2). MS (EI) m/z (\%): 489 (M+, 100). HRMS: $m / z$ (EI) for $\mathrm{C}_{25} \mathrm{H}_{19} \mathrm{~N}_{3} \mathrm{~S}_{4}$; calcd 489.0462; found: 489.0465.

\section{References}

1. Zyss D. S. In: Non linear optical properties of organic molecules and crystals, Vol 1 and 2; Academic Press: Orlando, 1987.

2. Prasad P. N. and Williams D. J. In: Introduction to non linear optical efects in molecules and polymers, John Wiley: New York, 1991.

3. Brosshard C.; Sutter K.; Petre P.; Hulliger J.; Florsheimer M.; Kaatz M.; Gunter P. In: Organic non-linear optical materials, Gordon and Breach Science Publishers: Amsterdam, 1995.

4. Mignani G.; Leising F.; Meyrueix R.; Samson M., Tetrahedron Lett. 1990, 31 (33), 4743.

5. Effenberger F.; Wuerthner F., Angew. Chem. 1993, $105,742$.

6. Effenberger F.; Wuerthner F., Angew. Chem. Int. Ed. Eng. 1993, 32, 719.

7. Rao V. P; Jen A. K.-Y.; Wong K. Y.; Drost K. J., J. Chem. Soc. Chem. Commun. 1993, 1118.

8. Rao V. P; Jen A. K-Y.; Wong K. Y.; Drost K. J., Tetrahedron Lett. 1993, 34, (11), 1747.

9. Rao V. P; Cai Y. M.; Jen A. K.-Y., J. Chem. Soc. Chem. Commun. 1994, 1689. 
10. Jen A. K.-Y.; Rao V. P; Drost K. J.; Wong K. Y.; Cava M. P., J. Chem. Soc. Chem. Commun. 1994, 2057.

11. Rao V. P; Wong K. Y.; Jen A. K.-Y.; Drost K. J., Chem. Mater. 1994, 6, 2210.

12. Wong K. Y.; Jen A. K.-Y.; Rao V. P; Drost K. J., Appl. Phys. B 1995, 61, 191.

13. Hutchins M. G.; Ferguson I.; McGeein D. J.; Morley J. O.; Ziss J.; Ledoux I., J. Chem. Soc. Perkin Trans 2 1995, 171.

14. Effenberger F.; Wuerthner F.; Steybe F., J. Org. Chem. 1995, 60, 2082.

15. Bedworth P. V.; Cai Y.; Jen A.; Marder S. R., J. Org. Chem. 1996, 61, 2242.

16. Steybe F.; Effenberger F; Beckman S.; Kramer P.; Glania C.; Wortmann R., Chem. Phys. 1997, 219, 317.

17. Steybe F.; Effenberger F; Gubler U.; Bosshard C.; Gunter P., Tetrahedron 1998, 54, 8469.

18. Bauerle P. In: The Synthesis of Oligothiophenes, D. Fichou Ed. Handbook of Oligo- and Polythiophenes, Wiley-VCH: Weinheim, 1999; Cap. 3, pp 89-173.

19. Eckert K.; Schroder A.; Hartmann H., Eur. J. Org. Chem 2000, 1327.

20. Wuerthner F.; Effenberger F., Chem. Phys. 1993, 173, 305.

21. Tour J. M.; Wu R., Macromolecules 1992, 25, 1901.

22. Barbarella G.; Bongini A.; Zambianchi M., Macromolecules 1994, 27, 3039.

23. Barbarella G.; Zambianchi M., Tetrahedron 1994, 50, 11249.

24. Folli U.; Iarossi D.; Montorsi M.; Mucci A.; Schenetti L., J. Chem. Soc., Perkin Trans. 1 1995, 537.

25. Shabana R.; Galal A.; Mark H. B.; Zimmer H.; Gronowitz S.; Hoernfeldt A. B., Phosphorus Sulfur 1990, 48, 239.

26. Amer A.; Burkhardt A.; Nkansah A.; Shabana R.; Galal A.; Mark H. B.; Zimmer H. B., Phosphorus Sulfur 1989, 42, 63.

27. Sone T.; Umetsu Y.; Sato K., Bull. Chem. Soc. Jpn 1991, 64, 864.

28. Sone T.; Sato K.; Umetsu Y.; Yoshimo A.; Takahashi K., Bull. Chem. Soc. Jpn 1994, 67, 2187.

29. Freeman F.; Lu H.; Zeng Q.; Rodriguez and E., J. Org. Chem. 1994, 59, 3665.

30. Masquelin T.; Obrecht D., Tetrahedron Lett., 1994, 35, 9387.

31. Raposo M. M. M.; Kirsch G., Heterocycles 2001, 55 (8), 1487.

32. Raposo M. M. M.; Kirsch G., Tetrahedron 2003, 59, (26), 4891.

33. Prim D.; Kirsch G., J. Chem. Soc., Perkin Trans. 1 1994, 2603. 
34. Prim D.; Kirsch G; Leising F.; Mignani G., J. Heterocycl. Chem. 1994, 31, 1005.

35. Prim D.; Joseph D.; Kirsch G., Phosphorus, Sulfur and Silicon 1994, 91, 137.

36. Costa S. P. G.; Griffiths J.; Kirsch G.; Oliveira-Campos A. M. F., Anales de Quimica Int. Ed. 1998, 94, 186.

37. Stille J. K., Angew. Chem. Int. Ed. Engl. 1986, 25, 508.

38. Farina V.; Krishnamurphy V.; Scott W. J. In: The Stille Reaction, Trost B. M. Ed. Organic Reactions. John Wiley and Sons: New York, 1997; Coll Vol. 50, pp 1-54.

39. Tietze L. F. In: The Knoevenagel Reaction, Trost B. M. Ed. Comprehensive Organic Synthesis. Pergamond Press: Oxford, 1991; Coll Vol. 2, pp 358-359.

40. Gopinatham S.; Gopinatham C.; Gupta J., Indian J. Chem. 1974, 12, 623.

41. Waite J.; Papadopoulos M., J. Phys. Chem. 1990, 94 (16), 6244.

42. Reichardt C. In: Solvents and Solvents Effects in Organic Chemistry, 2nd Ed., VCH: Weinheim, 1988.

43. Kosower E. M. In: An Introduction to Physical Organic Chemistry, Wiley: New York, 1968.

44. Kamlet M. J.; Abboud J-L. M.; Abraham M. H.; Taft R. W., J. Org. Chem. 1983, $48,2877$.

45. Kamlet M. J.; Abboud J-L M.; Abraham M. H.; Taft R. W., J. Am. Chem. Soc.; 1977, $99,6027$.

\section{Acknowledgements}

Thanks are due to Foundation for Science and Technology (Portugal) for financial suport through IBQF (UM) and through FEDER, POCTI (ref. POCTI/QUI/37816/2001). 


\section{Captions}

Figure 1. Correlation between absorption wavenumbers $v_{\max }(11 d)$ and the $\pi^{*}$ scale according to Kamlet and Taft. Aliphatic and dipolar aprotic solvents $(\diamond)$, protic solvents $(\square)$, chlorinated solvents $(\Delta)$ and aromatic solvents $(O)$.

Table 1. Yields and UV-vis absorption spectra of the coupled donor-acceptor oligothiophenes 9-16.

* All the UV/vis spectra were run in ethanol.

Table 2. UV-vis absorption maxima of bithiophenes 10d, 11d, terthiophenes 12b, 13d, 15d and quaterthiophene 16d in various solvents in comparison with $\pi^{*}$ values by Kamlet and Taft. 44

Table 3. Correlation of UV-Vis absorption maxima of bithiophenes 10d, 11d, terthiophenes 12b, 13d, 15d and quaterthiophene 16d and solvent parameter $\pi^{* a}$.

a Applied solvents ( $\pi^{*}$ value): $n$-hexane (-0.08), ciclohexane $(0.00)$, diethyl ether $(0.27)$, dioxane $(0.55)$, ethyl acetate $(0.55)$, tetrahydrofuran $(0.58)$, acetone $(0.71)$, acetonitrile (0.75), dimethylformamide (0.88), ethanol (0.54), methanol $(0.60)$, chloroform (0.76), dichloromethane (0.82), toluene (0.54). b Intercept, slope, and correlation coefficient $r$ of the linear solvatation energy relationship. $c$ Without $n$ hexane and ciclohexane. 


\section{Figures}

Figure 1

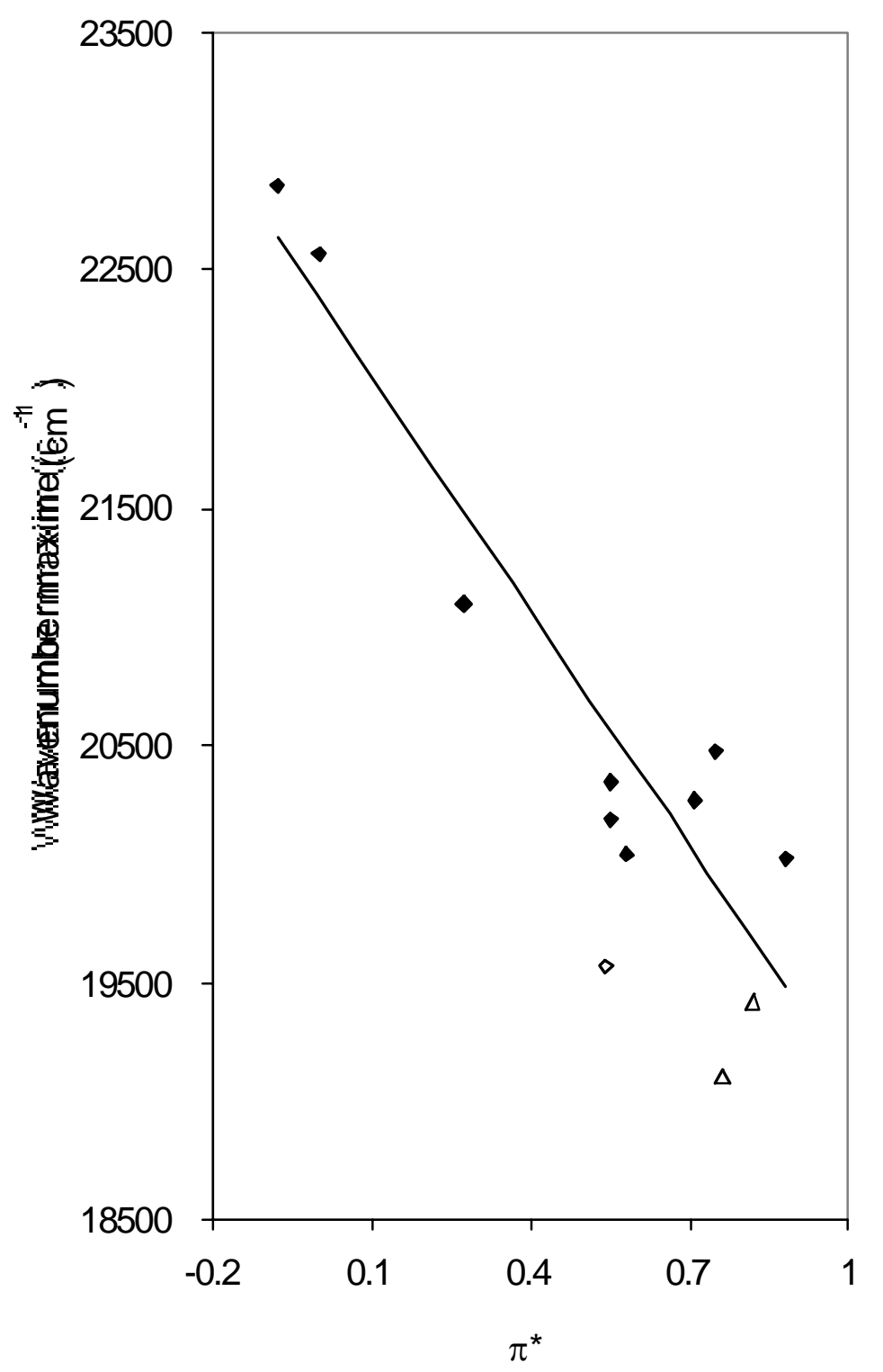




\section{Tables}

TABLE 1

\begin{tabular}{|c|c|c|c|c|c|c|}
\hline Entry & Bromide & $\begin{array}{l}\text { Bithienyl } \\
\text { stannane }\end{array}$ & Product & $\begin{array}{l}\text { Yield } \\
(\%)\end{array}$ & $\begin{array}{l}\text { Reaction } \\
\text { time }[h]\end{array}$ & $\begin{array}{c}\lambda \max ^{*}[\mathrm{~nm}] \\
(\varepsilon)\end{array}$ \\
\hline 1 & 1a & 8d & Piperidino-T2-4-CN-Ph 9d & 43 & 19 & $\begin{array}{l}420.0 \\
(18660)\end{array}$ \\
\hline 2 & $1 \mathbf{b}$ & $8 a$ & Methoxy- $\mathrm{T}_{2}-4-\mathrm{NO}_{2}-\mathrm{Ph} \mathbf{1 0 a}$ & 65 & 10.5 & $\begin{array}{l}413.0 \\
(25750)\end{array}$ \\
\hline 3 & $1 \mathbf{b}$ & $8 \mathbf{b}$ & $N, N$-Dimethyl- $\mathrm{T}_{2}-4-\mathrm{NO}_{2}-\mathrm{Ph} \mathbf{1 0 b}$ & 42 & 19 & $\begin{array}{l}461.0 \\
(10050)\end{array}$ \\
\hline 4 & $1 b$ & 8c & $N, N$-Diethyl- $\mathrm{T}_{2}-4-\mathrm{NO}_{2}-\mathrm{Ph} \mathbf{1 0 c}$ & 44 & 24.5 & $\begin{array}{c}474.5 \\
(16800)\end{array}$ \\
\hline 5 & $1 \mathbf{b}$ & 8d & Piperidino- $\mathrm{T}_{2}-4-\mathrm{NO}_{2}-\mathrm{Ph} \mathbf{1 0 d}$ & 53 & 8 & $\begin{array}{l}453.0 \\
(10000)\end{array}$ \\
\hline 6 & 1d & 8d & $\begin{array}{c}\text { Piperidino- } \mathrm{T}_{2}-4-\left[\mathrm{CH}=\mathrm{C}(\mathrm{CN})_{2}\right]-\mathrm{Ph} \\
\mathbf{1 1 d}\end{array}$ & 56 & 30 & $\begin{array}{l}468.0 \\
(21400)\end{array}$ \\
\hline 7 & $2 \mathbf{a}$ & $8 \mathbf{b}$ & $N, N$-Dimethyl-T3-CHO 12b & 46 & 20 & $\begin{array}{c}465.5 \\
(22690)\end{array}$ \\
\hline 8 & $2 \mathbf{a}$ & 8d & Piperidino-T3-CHO 13d & 51 & 17 & $\begin{array}{c}456.0 \\
(15260)\end{array}$ \\
\hline 9 & $2 \mathbf{b}$ & 8d & Piperidino- $\mathrm{T}_{3}-\mathrm{NO}_{2} \mathbf{1 4 d}$ & 53 & 33 & $\begin{array}{c}504.0 \\
(10100)\end{array}$ \\
\hline 10 & 2c & 8d & Piperidino- $\mathrm{T}_{3}-\left[\mathrm{CH}=\mathrm{C}(\mathrm{CN})_{2}\right] \mathbf{1 5 d}$ & 55 & 33.5 & $\begin{array}{c}545.5 \\
(23770)\end{array}$ \\
\hline 11 & 6 & 8d & Piperidino- $\mathrm{T}_{4}-\left[\mathrm{CH}=\mathrm{C}(\mathrm{CN})_{2}\right] \mathbf{1 6 d}$ & 45 & 30 & $\begin{array}{l}510.5 \\
(12000)\end{array}$ \\
\hline
\end{tabular}




\section{Table 2}

\begin{tabular}{|c|c|c|c|c|c|c|c|c|c|c|c|c|c|}
\hline Solvents & $\pi^{*}$ & $\begin{array}{r}\quad 1 \\
\lambda_{\max } \\
{[\mathrm{nm}]}\end{array}$ & $\begin{array}{l}\text { 0d } \\
v_{\max } \\
{\left[\mathrm{cm}^{-1}\right]}\end{array}$ & $\begin{array}{r}\quad 11 \\
\lambda_{\max } \\
{[\mathrm{nm}]}\end{array}$ & $\begin{array}{l}1 \text { d } \\
v_{\max } \\
{\left[\mathrm{cm}^{-1}\right]}\end{array}$ & $\begin{array}{r}\quad 12 \\
\lambda_{\max } \\
{[\mathrm{nm}]}\end{array}$ & $\begin{array}{l}2 \mathbf{b} \\
u_{\max } \\
{\left[\mathrm{cm}^{-1}\right]}\end{array}$ & $\begin{array}{r}1 \\
\lambda_{\max } \\
{[\mathrm{nm}]}\end{array}$ & $\begin{array}{l}3 d \\
U_{\max } \\
{\left[\mathrm{cm}^{-1}\right]}\end{array}$ & $\begin{array}{r}1 \\
\lambda_{\max } \\
{[\mathrm{nm}]}\end{array}$ & $\begin{array}{l}\mathbf{5 d} \\
v_{\max } \\
{\left[\mathrm{cm}^{-1}\right]}\end{array}$ & $\begin{array}{l}\quad 16 \\
\lambda_{\max } \\
{[\mathrm{nm}]}\end{array}$ & $\begin{array}{l}\mathbf{6 d} \\
U_{\max } \\
{\left[\mathrm{cm}^{-1}\right]}\end{array}$ \\
\hline$n$-hexane & -0.08 & 441.5 & 22650 & 437.5 & 22857 & 443.0 & 22573 & 437.5 & 22857 & 532.5 & 18779 & & -- \\
\hline ciclohexane & 0.00 & 446.5 & 22396 & 443.0 & 22573 & 448.0 & 22321 & 443.0 & 22573 & 539.0 & 18552 & & -- \\
\hline diethyl ether & 0.27 & 448.5 & 22296 & 474.0 & 21097 & 452.0 & 22123 & 444.0 & 22522 & 538.0 & 18587 & 502.0 & 19920 \\
\hline dioxane & 0.55 & 455.0 & 22471 & 495.5 & 20181 & 458.0 & 21834 & 453.0 & 22075 & 539.5 & 18535 & 520.0 & 19230 \\
\hline ethyl acetate & 0.55 & 454.0 & 22026 & 491.5 & 20345 & 457.0 & 21881 & 450.0 & 22222 & 538.5 & 18570 & 513.0 & 19493 \\
\hline tetrahydrofuran & 0.58 & 459.5 & 21762 & 499.0 & 20040 & 461.5 & 21668 & 454.5 & 22002 & 548.0 & 18248 & 521.0 & 19193 \\
\hline acetone & 0.71 & 458.5 & 21810 & 493.5 & 20263 & 461.5 & 21668 & 454.5 & 22002 & 544.5 & 18365 & 515.0 & 19417 \\
\hline acetonitrile & 0.75 & 457.0 & 21881 & 488.5 & 20470 & 462.5 & 21621 & 453.0 & 22075 & 542.0 & 18450 & 503.0 & 19880 \\
\hline dimethylformamide & 0.88 & 470.5 & 21253 & 499.5 & 20020 & 471.0 & 21231 & 463.0 & 21598 & 555.5 & 18001 & 526.5 & 18993 \\
\hline ethanol & 0.54 & 453.0 & 22075 & 468.0 & 21367 & 465.5 & 21482 & 456.0 & 21929 & 545.5 & 18331 & 510.5 & 19588 \\
\hline methanol & 0.60 & 450.0 & 22222 & 468.5 & 21344 & 464.5 & 21528 & 454.5 & 22002 & 539.0 & 18552 & 504.5 & 19821 \\
\hline chloroform & $0.58 / 0.76^{45}$ & 457.0 & 21881 & 523.5 & 19102 & 470.5 & 21253 & 468.0 & 21367 & 568.5 & 17590 & 538.0 & 18587 \\
\hline dichloromethane & 0.82 & 467.5 & 21390 & 515.0 & 19417 & 469.0 & 21321 & 462.5 & 21621 & 562.5 & 17777 & 528.0 & 18939 \\
\hline
\end{tabular}


TABLE 3

\begin{tabular}{cccc}
\hline & & Regression analysis \\
Compounds & $v_{0}\left[\mathrm{~cm}^{-1}\right]$ & $\left.\mathrm{cm}^{-1}\right]$ & $r^{\mathrm{b}}$ \\
\hline 10d & 22588 & -1120 & -0.8084 \\
11d & 22376 & -3297 & -0.8495 \\
12d & 22415 & -1294 & -0.9410 \\
13d & 22730 & -1252 & -0.9037 \\
15d & 18698 & -1003 & -0.8869 \\
16dc & 20019 & -1002 & -0.9150 \\
\hline
\end{tabular}




\section{Schemes}<smiles>Brc1cccs1</smiles>

3<smiles>N#CC(C#N)=Cc1ccc(-c2ccc(Br)s2)s1</smiles>

6 i, $n$-BuLi, dry ether, $0{ }^{\circ} \mathrm{C}, \mathrm{Ar}$

ii, $(n-\mathrm{Bu})_{3} \mathrm{SnCl}$, dry ether, $-78{ }^{\circ} \mathrm{C}, \mathrm{Ar}$

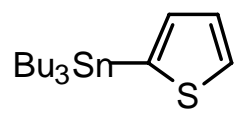

4

2c, $\mathrm{Pd}\left(\mathrm{PPh}_{3}\right)_{4}$, toluene, $80{ }^{\circ} \mathrm{C}, \mathrm{Ar}$

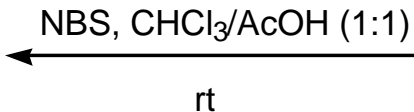

Scheme 1

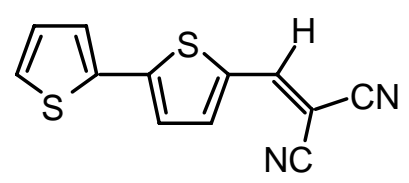

5 


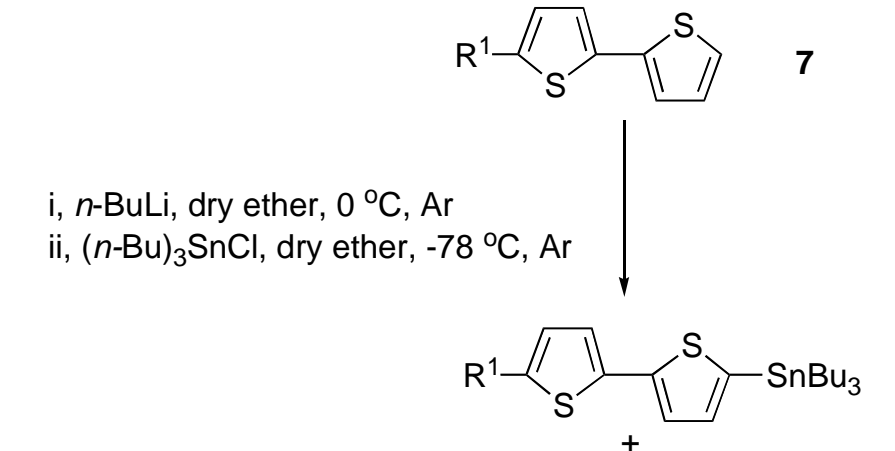

$\mathrm{Pd}\left(\mathrm{PPh}_{3}\right)_{4}$, toluene,

$80{ }^{\circ} \mathrm{C}, \mathrm{Ar}$
8 a $\mathrm{R}^{1}=\mathrm{OMe}$

b $\mathrm{R}^{1}=\mathrm{NMe}_{2}$

c $\mathrm{R}^{1}=\mathrm{NEt}_{2}$

d $\mathrm{R}^{1}=$ piperidino

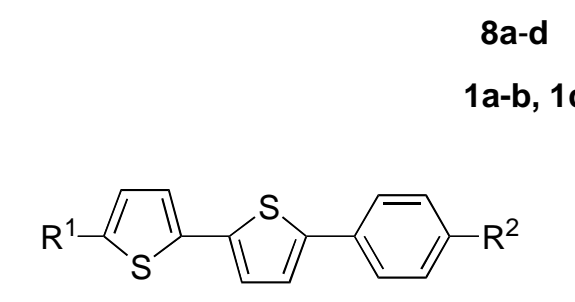

9d $\mathrm{R}^{1}=$ piperidino, $\mathrm{R}^{2}=\mathrm{CN}$

10a $\mathrm{R}^{1}=\mathrm{OMe}, \mathrm{R}^{2}=\mathrm{NO}_{2}$

10b $\mathrm{R}^{1}=\mathrm{NMe}_{2}, \mathrm{R}^{2}=\mathrm{NO}_{2}$

10c $\mathrm{R}^{1}=\mathrm{NEt}_{2}, \mathrm{R}^{2}=\mathrm{NO}_{2}$

10d $R^{1}=$ piperidino, $\mathrm{R}^{2}=\mathrm{NO}_{2}$

11d $\mathrm{R}^{1}=$ piperidino, $\mathrm{R}^{2}=\mathrm{CH}=\mathrm{C}(\mathrm{CN})_{2}$
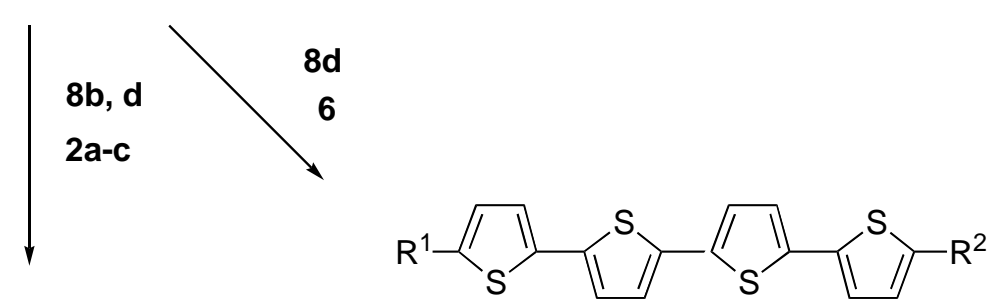

16d $\mathrm{R}^{1}=$ piperidino, $\mathrm{R}^{2}=\mathrm{CH}=\mathrm{C}(\mathrm{CN})_{2}$ 12b $\mathrm{R}^{1}=\mathrm{NMe}_{2}, \quad \mathrm{R}^{2}=\mathrm{CHO}$

13d $\mathrm{R}^{1}=$ piperidino, $\mathrm{R}^{2}=\mathrm{CHO}$

14d $\mathrm{R}^{1}=$ piperidino, $\mathrm{R}^{2}=\mathrm{NO}_{2}$

15d $\mathrm{R}^{1}=$ piperidino, $\mathrm{R}^{2}=-\mathrm{CH}=\mathrm{C}(\mathrm{CN})_{2}$
Scheme 2 